\title{
BARS AND BLACK HOLES
}

\author{
ERIC EMSELLEM
}

ESO

\section{Introduction}

Massive black holes are now thought to be present at the centre of a fair fraction of nearby galaxies. The origin of these central dark masses is still unknown, although tentative explanations have been proposed in an attempt to reconcile non-active galaxies with AGNs and quasars. The activity of a galaxy may then mostly depend on the efficiency of gas accretion onto the central dark object. It is important to note that many of the galaxies which are today candidates for the presence of a massive black hole are spirals. In this context, bars should play an important role in the evolution (and formation?) of a central mass, since (1) they are present in a significant fraction of spirals, (2) they may be efficient drivers of gas accretion. If indeed most of present day galaxies hosts a central dark mass, then bars and black holes should coexist in a significant fraction of them. We examine here the cases of 3 edge-on galaxies which are candidates for the presence of a central black holes: NGC 4570, NGC 3115 and M 104.

\section{3 cases}

\subsection{NGC 4570}

NGC 4570 is an S0 galaxy with $M_{B} \sim-19$. Frank van den Bosch and I discovered the presence of two double peaks symmetric with respect to the centre which we interpreted as 2 systems of rings. Assuming the inner peaks correspond to the Inner Linblad Resonance of a tumbling figure, we can predict the locations of the other resonances using a Multi-Gaussian Expansion mass model of NGC 4570. We thus found that the second ring system is nearly coincident with the predicted Ultra Harmonic Resonance. Furthermore, the $U-V$ and $V-I$ colour profiles as well as the kinematics, 
show significant changes at the locations of the main resonances (ILR, UHR, CR). This strongly suggests that indeed NGC 4570 was shaped under the influence of a tumbling potential (a bar). In this context, we propose that the double disk structures (inner disk and a Freeman type II outer disk) seen in NGC 4570 and more that $50 \%$ of edge-on S0 galaxies are the remnants of a bar driven secular evolution. The inner disc of NGC 4570 appears young $(<2$ Gyrs) and metal rich, which suggests that a significant mass was recently accreted within the central $50 \mathrm{pc}$ of the galaxy.

\subsection{NGC 3115}

Emsellem \& Dejonghe recently built 2 and 3 integral models of NGC 3115 which confirm the presence of a central dark mass of about $910^{8} \mathrm{M}_{\odot}$. NGC 3115 also contains a double disc structure, reminiscent of the presence of a tumbling potential, as well as a weak spiral arm starting at the end of the inner disc. In many aspects, NGC 3115 is qualitatively similar to NGC 4570 although the scales are different (NGC 3115: $M_{B} \sim-20.13$ ). As in NGC 4570, there is no detected emitting gas. I propose that NGC 3115 also belong to the class of galaxies shaped under the influence of a bar. In the case of NGC 3115, there are also indications of a past merger event.

\subsection{104}

It has already been shown that the double disc structure and the rings in the Sombrero galaxy do suggest the presence of a (dissolved?) bar (e.g. Emsellem et al. 1996, A\&A 312, 777). This galaxy is also an excellent candidate for the presence of a central dark object (e.g. Kormendy et al. 1997, ApJL 473, 91). Although there are strong similarities between NGC 4570, NGC 3115 and the Sombrero galaxy, the latter contains a significant fraction of gas in the outer part (the OLR ring) and in the inner $2 \mathrm{kpc}$ (the UHR ring and the inner gas disc). M 104 may therefore still be in the process of central gas accretion, which is supported by the observed activity of its nucleus. This hypothesis is strenghtened by recent TIGER data which reveal strongly non-circular motions in the inner 2 arcseconds $(\sim 100 \mathrm{pc})$.

\section{Conclusions}

I discussed here the hypothesis of NGC 4570, NGC 3115, and M 104 having been shaped under the influence of a tumbling triaxial potential. Kinematics and dynamical models show that these three galaxies may contain a central dark mass $\left(M_{d m} \sim 3.5,9\right.$ and $1010^{8} \mathrm{M}_{\odot}$ respectively). The next issue to be addressed is then: what fraction of this mass was accreted due to the bar phase in these galaxies, and when? 